\title{
Grisaille automnale, espoir printanier
}

Roger Ladouceur MD MSc CCMF(SP) FCMF, RÉDACTEUR SCIENTIFIQUE ADJOINT

$\mathrm{N}$ oël s'en vient. C'est l'occasion d'écrire quelque chose de réconfortant, un message d'espoir...

Je veux bien, moi, mais je ne trouve pas que ce que nous vivons actuellement soit une période particulièrement réconfortante. Cette maladie qui se propage comme le feu aux poudres. Des millions de personnes infectées à travers le monde. Des milliers de morts. Au moment d'écrire ces lignes, on dénombrait plus de 52000 personnes infectées et 11000 décès au Canada ${ }^{1}$; et, respectivement, près de 57 millions et 1,4 million à travers le monde ${ }^{2}$. Parmi les régions particulièrement touchées, des nations occidentales:la France, l'Espagne, l'Italie et la Russie, sans parler des États-Unis et... du Québec et, au cours des dernières semaines, la plupart des provinces canadiennes.

Pas de quoi se réjouir!

Et toutes ces contraintes. Les restaurants, les bars, les cinémas, les salles de spectacle, les piscines, les gyms: tous fermés. Les couvre-feux. Fini les grands rassemblements et même les petits. Pas plus que six personnes ensemble. "Restez chez vous!", qu'on clame à répétition. Et toujours pas de traitement efficace, et l'attente désespérée d'un ou plusieurs vaccins.

Non, pas de quoi se réjouir!

Et parallèlement, les troubles de santé mentale qui se propagent au même rythme que la pandémie. Des gens qui n'osent plus sortir de chez eux. Des gens qui paniquent, qui angoissent. Des personnes âgées qui ne peuvent plus quitter leur appartement. Des gens qui passent leurs journées chez eux. À ne rien faire. À suivre des séries télévisées. À regarder Breaking $\mathrm{Bad}^{3}$. Pas très drôle, cette série - je n'ai pas ri une seule fois - évidemment, ce n'était pas le but. Ou pire, La Servante écarlate ${ }^{4}$ d'après l'œuvre de Margaret Atwood. Épouvantable. Absolument épouvantable. Le chaos social. Cela pourrait-il arriver un jour? Avec les élections américaines en toile de fond.

Même Mr Bean 5 m'apparaît morose... C'est tout dire!

Évidemment, cela pourrait être pire. Bien pire en réalité. Il suffit de lire Suite française ${ }^{6}$ ou Les Bienveillantes ${ }^{7}$ pour s'en convaincre. Ce que nous vivons n'a rien à voir avec les conflits, les guerres et les cataclysmes naturels. Au moins, actuellement, nous pouvons nous nourrir, nous loger, nous chauffer et communiquer. Dans la pyramide des besoins de Maslow, nous ne sommes pas si pires. ${ }^{8}$

Et là, tout à coup, sans raison apparente, me vient l'histoire de C't'à ton tour, Laura Cadieux,' de Michel Tremblay.

L'histoire d'une femme déprimée et obsédée par son poids qui va régulièrement chez son médecin pour ses injections. Chemin faisant, elle perd son fils dans le métro. Pendant qu'elle accourt chez le médecin et que sa meilleure amie fouille la ville pour retrouver le fils manquant, lequel est en sécurité à la maison, Laura finit par arriver chez son médecin. La salle d'attente est pleine. Toujours les mêmes personnes qui se plaignent de leurs bobos. Laura quitte sans avoir été vue par le médecin. Elle reviendra un autre jour!

C'était l'époque où les médecins de famille étaient au centre de la vie de bien des gens. C'est encore le cas aujourd'hui. Le médecin de famille demeure l'un des seuls professionnels de la santé qui peut s'occuper de tous les habitants de son village ou de son quartier: les enfants, les adultes et les personnes âgées; capable de les accompagner tout au long de la vie, de la naissance à la mort; sachant évaluer leurs malaises, leurs mal-êtres et leurs maladies; capable d'en déterminer la gravité; sachant quand investiguer ou référer, si nécessaire. Mais surtout l'un des seuls qui peut être présent et qui sait écouter, encourager, soutenir et rassurer. Particulièrement en cette période trouble. Pas surprenant que tant de personnes soient à la recherche d'un médecin de famille ou chérissent le leur.

Me reviennent alors les paroles de Christophe Maé dont nous devrions nous rappeler quand nous voyons nos patients, inquiets et mal en point:

Il est où le bonheur? Il est où?

Il est là ${ }^{10}$

Après tout, cela pourrait être bien pire.

Déjà que, au moment d'écrire ces lignes, on a annonce la découverte de 3 vaccins efficaces contre la COVID-19.

Allez, ça ira mieux.

\section{Références}

1. Santé Infobase Canada. Maladie à coronavirus de 2019 (COVID-19): mise à jour quotidienne sur l'épidémiologie. Ottawa, ON: Gouvernement du Canada; 2020. Accessible à: https://sante-infobase.canada.ca/covid-19/resume-epidemiologique-cas-covid-19. html. Réf. du 20 novembre 2020.S

2. Center for Systems Science and Engineering. COVID-19 dashboard. Baltimore, MD: Johns Hopkins University; 2020. Accessible à: https://coronavirus.jhu.edu/map.html. Réf. du 20 novembre 2020 .

3. Gilligan V. Breaking bad. New York, NY: AMC Network Entertainment; 2008.

4. Miller B. The handmaid's tale. Beverly Hills, CA: MGM Television; 2017.

5. Atkinson R, Curtis R. Mr Bean. London, R.-U.: Tiger Aspect Productions; 1990.

6. Némirovsky I. Suite française. Paris, France: Éditions Denoël; 2004.

7. Littell J. Les Bienveillantes. Paris, France: Gallimard; 2006.

8. Wikipedia [encyclopédie en ligne]. Pyramide des besoins. Los Angeles, CA: Wikipedia Foundation Ltd; 2020. Accessible à: https://fr.wikipedia.org/wiki/Pyramide_des_ besoins. Réf. du 24 novembre 2020.

9. Tremblay M. C't'à ton tour, Laura Cadieux. Montréal, QC: Bibliothèque québécoise; 1997. 10. FrenchVariety. Christophe Mae - Il est où le bonheur (version avec choeurs) [vidéo]. YouTube; 2019. Accessible à : https://www.youtube.com/watch?v=whjM_WaWbFI. Réf. du 24 novembre 2020. 